\title{
Crystal structure of aquabis(1,10-phenanthroline $)-\mu-\left(1,1^{\prime}\right.$-biphenyl-2,2'- dicarboxylato)dinitratodilead(II), $\mathrm{Pb}_{2}\left(\mathrm{C}_{12} \mathrm{H}_{8} \mathrm{~N}_{2}\right)_{2}\left(\mathrm{C}_{14} \mathrm{H}_{8} \mathrm{O}_{4}\right)\left(\mathrm{H}_{2} \mathrm{O}\right)\left(\mathrm{NO}_{3}\right)_{2}$
}

\author{
Q. Miao*, Y.-J. Zhao, Y.-Q. Cheng and M.-L. Hu
}

Wenzhou Normal College, Department of Chemistry and Material Science, Wenzhou, 325027 P. R. China

Received January 13, 2005, accepted and available on-line February 7, 2005; CCDC no. 1267/1459
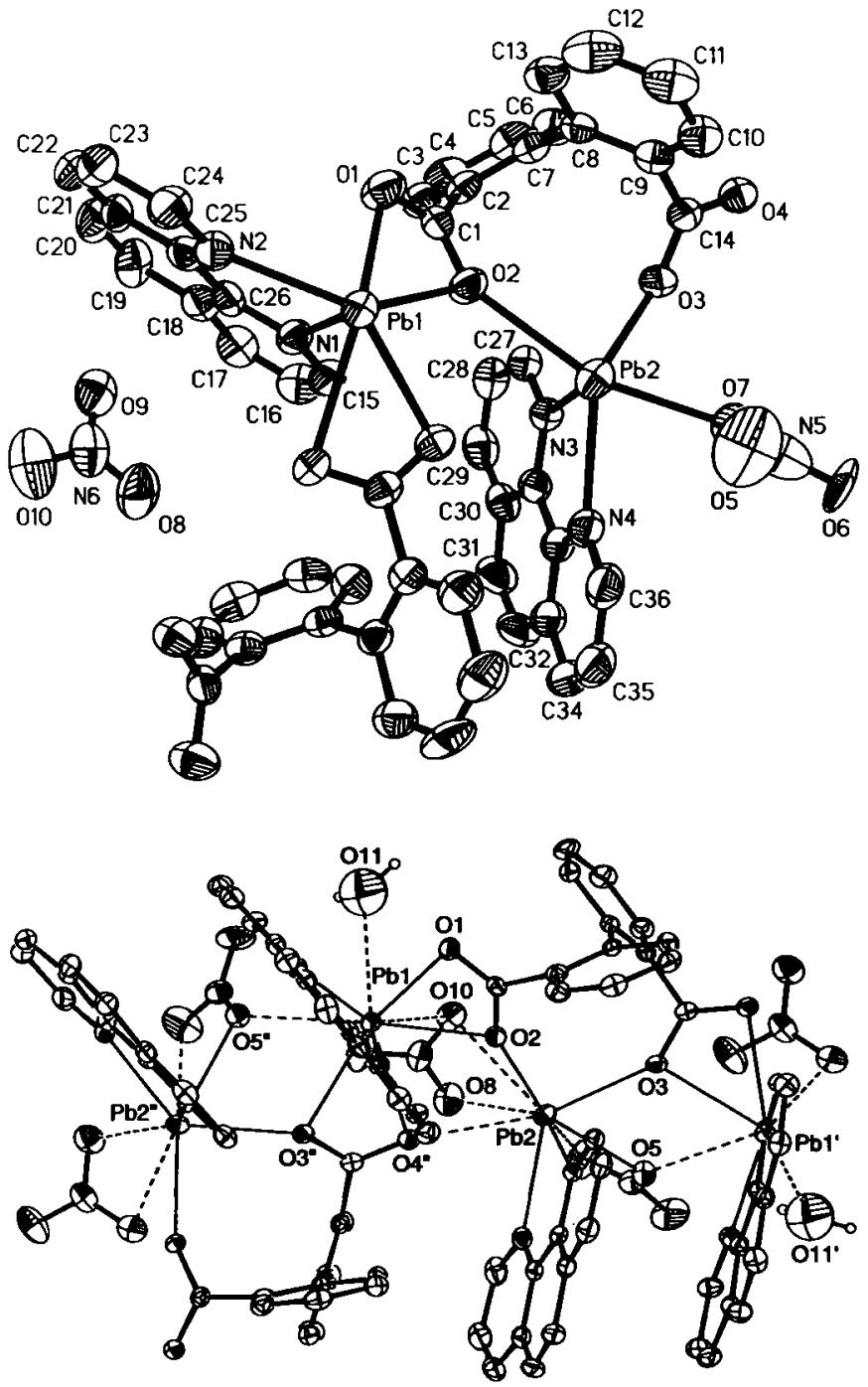

Abstract

$\mathrm{C}_{38} \mathrm{H}_{26} \mathrm{~N}_{6} \mathrm{O}_{11} \mathrm{~Pb}_{2}$, monoclinic, $P 121 / c 1$ (no. 14),

$a=10.8980(7) \AA, b=23.449$ (2) $\AA, c=14.814(1) \AA$,

$\beta=110.57(3)^{\circ}, V=3544.3 \AA^{3}, Z=4$,

$R_{\mathrm{gt}}(F)=0.029, w R_{\mathrm{ref}}\left(F^{2}\right)=0.070, T=298 \mathrm{~K}$.

\section{Source of material}

The title compound was synthesized with the hydrothermal method from a mixture of $2,2^{\prime}$-biphenyldicarboxylate $(2 \mathrm{mmol}$, $0.48 \mathrm{~g}$ ), $\mathrm{Pb}\left(\mathrm{NO}_{3}\right)_{2}$ (1 mmol, $\left.0.66 \mathrm{~g}\right), 1,10$-phenanthroline ( $2 \mathrm{mmol}$,

$0.36 \mathrm{~g})$ and water $(20 \mathrm{~mL})$ in a $30 \mathrm{~mL}$ Teflon-lined stainless-steel reactor. The solution was heated to $430 \mathrm{~K}$ for three days. After the reaction system was slowly cooled to room temperature, then the yellow block crystals were collected and washed with distilled water (yield $65 \%$ ).

\section{Discussion}

Lead(II) is capable of exhibiting variable coordination with or without stereochemically active lone pair of electrons [1]. The absence of crystal field stabilization energy effects also allows the $\mathrm{Pb}$ (II) cations to adopt different coordinations not restricted to octahedral, tetrahedral or square planar [2]. Of these, a number of polymeric $\mathrm{Pb}$ (II) compounds have been structurally characterized [3-5]. Carboxylate anions possess versatile binding modes to metal cations [6], therefore, metal complexes with carboxylate anions $[7,8]$ have been extensively studied, including several $4,4^{\prime}$-biphenyldicarboxylate complexes $[9,10]$. However, the number of complexes constructed from $2,2^{\prime}$-biphenyldicarboxylate anions and metal cations are still limited [11]. Thus, we have selected the $\mathrm{Pb}$-bpdc-phen (bpdc $=1,1^{\prime}$-biphenyl-2,2' -dicarboxylate dianion and phen $=1,10$-phenanthroline) system to extend this area of research and present here the crystal structure of the title compound, $\left.\left[\mathrm{Pb}_{2} \text { (bpdc)(phen) }\right)_{2}\left(\mathrm{NO}_{3}\right)_{2}\left(\mathrm{H}_{2} \mathrm{O}\right)\right]_{n}$ (figure, top). There are two different kinds of independent $\mathrm{Pb}$ centres bridged by bpdc ligands to form a novel catena chain (figure, bottom). The $\mathrm{Pb} 2$ centre has a deformed trigonal-bipyramidal environment completed by two $\mathrm{O}$ atoms belonging to two carboxylate of a bpdc ligand, two $\mathrm{N}$ atoms from a phen molecule and one nitrate $\mathrm{O}$ atom. The $\mathrm{Pbl}$ centre is irregularly six-coordinated by four carboxylate $\mathrm{O}$ atoms from two bpdc dianions and two $\mathrm{N}$ atoms from a phen ligand. $\mathrm{All} \mathrm{Pb}-\mathrm{O}_{\text {carboxylate }}$ bond lengths are within the range of $2.555(4) \AA-2.700(4) \AA$, being typical for such distances [2]. The $\mathrm{Pb}-\mathrm{N}$ bond lengths of 2.512(5) $\AA-2.609(6) \AA$ are also in the range of $\mathrm{Pb}-\mathrm{N}_{\text {phen }}$ bond distances observed for $\mathrm{re}$ lated structures $(2.540(4) \AA-2.599(3) \AA[12,13])$. The phen ligand and two carboxylate groups of different bpdc dianions are in chelating and two carboxylato $O$ atoms from two bpdc dianions are in bridging modes.

Table 1. Data collection and handling.

\section{Crystal:}

Wavelength:

$\mu$ :

Diffractometer, scan mode:

$2 \theta_{\max }$ :

$N(h k l)_{\text {measured, }} N(h k l)_{\text {maique: }}$

Criterion for $I_{\mathrm{obs}}, N(h k l)_{\mathrm{gt}}$ :

$N($ param hefined:

Programs: yellow block, size $0.08 \times 0.20 \times 0.34 \mathrm{~mm}$ Mo $K_{\alpha}$ radiation $(0.71073 \AA)$ $95.62 \mathrm{~cm}^{-1}$

Bruker APEX CCD, 600 frames, $\Delta \omega=0.3^{\circ}$ $50.5^{\circ}$

18805,6376

$I_{\mathrm{obs}}>2 \sigma\left(I_{\mathrm{obs}}\right), 5124$

515

SHEL X-97 [14], ORTEP-II [15]

\footnotetext{
* Correspondence author (e-mail: zkrist2005@yahoo.com.cn)
} 
Table 2. Atomic coordinates and displacement parameters (in $\AA^{2}$ ).

\begin{tabular}{llllll}
\hline Atom & Site & $x$ & $y$ & $z$ & $U_{\text {iso }}$ \\
\hline H(3) & $4 e$ & 0.5877 & 0.3529 & 0.7286 & 0.061 \\
H(4) & $4 e$ & 0.7225 & 0.3530 & 0.8879 & 0.069 \\
H(5) & $4 e$ & 0.6312 & 0.3614 & 1.0075 & 0.062 \\
H(6) & $4 e$ & 0.4087 & 0.3686 & 0.9665 & 0.059 \\
H(10) & $4 e$ & -0.0492 & 0.3206 & 0.8066 & 0.061 \\
H(11) & $4 e$ & -0.1382 & 0.4034 & 0.7278 & 0.073 \\
H(12) & $4 e$ & -0.0098 & 0.4675 & 0.6860 & 0.074 \\
H(13) & $4 e$ & 0.2116 & 0.4522 & 0.7299 & 0.059 \\
H(15) & $4 e$ & 0.4533 & 0.2463 & 0.5472 & 0.056 \\
H(16) & $4 e$ & 0.6769 & 0.2336 & 0.6068 & 0.065 \\
H(17) & $4 e$ & 0.8107 & 0.3057 & 0.5904 & 0.061 \\
H(19) & $4 e$ & 0.8329 & 0.4026 & 0.5252 & 0.069 \\
H(20) & $4 e$ & 0.7392 & 0.4812 & 0.4414 & 0.067 \\
H(22) & $4 e$ & 0.5346 & 0.5316 & 0.3390 & 0.068 \\
\hline
\end{tabular}

Table 2. Continued.

\begin{tabular}{llllll}
\hline Atom & Site & $x$ & $y$ & $z$ & $U_{\text {iso }}$ \\
\hline H(23) & $4 e$ & 0.3097 & 0.5334 & 0.2705 & 0.070 \\
H(24) & $4 e$ & 0.1896 & 0.4600 & 0.3027 & 0.060 \\
H(27) & $4 e$ & 0.4347 & 0.2445 & 0.7821 & 0.049 \\
H(28) & $4 e$ & 0.6541 & 0.2213 & 0.8295 & 0.060 \\
H(29) & $4 e$ & 0.7162 & 0.1370 & 0.7760 & 0.067 \\
H(31) & $4 e$ & 0.6498 & 0.0457 & 0.6845 & 0.074 \\
H(32) & $4 e$ & 0.4912 & -0.0111 & 0.5871 & 0.079 \\
H(34) & $4 e$ & 0.2525 & -0.0235 & 0.4805 & 0.089 \\
H(35) & $4 e$ & 0.0445 & 0.0133 & 0.4181 & 0.087 \\
H(36) & $4 e$ & -0.0008 & 0.0981 & 0.4805 & 0.075 \\
(e) & $4 e$ & $-0.022(1)$ & $0.5605(7)$ & $0.570(1)$ & $0.233(5)$ \\
H(11A) & $4 e$ & $-0.03(1)$ & $0.542(6)$ & $0.518(6)$ & 0.280 \\
H(11B) & $4 e$ & $0.055(8)$ & $0.566(7)$ & $0.621(7)$ & 0.280 \\
& & & & & \\
& & & & &
\end{tabular}

Table 3. Atomic coordinates and displacement parameters (in $\AA^{\mathbf{2}}$ ).

\begin{tabular}{|c|c|c|c|c|c|c|c|c|c|c|}
\hline Atom & Site & $x$ & $y$ & $z$ & $U_{11}$ & $U_{22}$ & $U_{33}$ & $U_{12}$ & $U_{13}$ & $U_{23}$ \\
\hline$P b(1)$ & $4 e$ & $0.20278(2)$ & $0.338966(9)$ & $0.43820(2)$ & $0.0379(1)$ & $0.0370(1)$ & $0.0408(1)$ & $-0.00133(9)$ & $0.0138(1)$ & $0.00301(9)$ \\
\hline$P b(2)$ & $4 e$ & $0.13434(2)$ & $0.215179(9)$ & $0.62939(2)$ & $0.0357(1)$ & $0.0395(1)$ & $0.0492(2)$ & $0.00148(9)$ & $0.0102(1)$ & $0.0080(1)$ \\
\hline $\mathbf{N}(1)$ & $4 e$ & $0.4495(4)$ & $0.3207(2)$ & $0.4893(3)$ & $0.040(3)$ & $0.037(3)$ & $0.040(3)$ & $-0.004(2)$ & $0.013(2)$ & $-0.002(2)$ \\
\hline $\mathbf{N}(2)$ & $4 e$ & $0.3355(5)$ & $0.4141(2)$ & $0.3837(3)$ & $0.044(3)$ & $0.041(3)$ & $0.043(3)$ & $0.000(2)$ & $0.016(3)$ & $0.002(2)$ \\
\hline $\mathbf{N}(3)$ & $4 e$ & $0.3656(4)$ & $0.1789(2)$ & $0.6990(3)$ & $0.038(3)$ & $0.035(3)$ & $0.036(3)$ & $-0.001(2)$ & $0.014(2)$ & $0.001(2)$ \\
\hline $\mathbf{N}(4)$ & $4 e$ & $0.1761(5)$ & $0.1153(2)$ & $0.5679(3)$ & $0.060(4)$ & $0.047(3)$ & $0.038(3)$ & $-0.016(3)$ & $0.019(3)$ & $-0.003(2)$ \\
\hline$N(5)$ & $4 e$ & $-0.0431(6)$ & $0.1228(3)$ & $0.6881(5)$ & $0.056(4)$ & $0.058(4)$ & $0.075(5)$ & $-0.007(3)$ & $0.022(4)$ & $-0.003(3)$ \\
\hline$N(6)$ & $4 e$ & $0.9298(6)$ & $0.2762(3)$ & $0.4339(5)$ & $0.037(4)$ & $0.097(5)$ & $0.070(5)$ & $0.008(3)$ & $0.021(4)$ & $0.020(4)$ \\
\hline$O(1)$ & $4 e$ & $0.3151(5)$ & $0.3962(2)$ & $0.5920(3)$ & $0.088(4)$ & $0.042(2)$ & $0.039(2)$ & $-0.015(2)$ & $0.015(2)$ & $-0.001(2)$ \\
\hline$O(2)$ & $4 e$ & $0.2799(4)$ & $0.3065(2)$ & $0.6196(3)$ & $0.055(3)$ & $0.038(2)$ & $0.041(2)$ & $-0.016(2)$ & $0.014(2)$ & $-0.006(2)$ \\
\hline$O(3)$ & $4 e$ & $0.2178(4)$ & $0.2432(2)$ & $0.8148(3)$ & $0.048(2)$ & $0.032(2)$ & $0.048(2)$ & $0.006(2)$ & $0.020(2)$ & $0.000(2)$ \\
\hline O(4) & $4 e$ & $0.1977(4)$ & $0.2721(2)$ & $0.9509(3)$ & $0.041(2)$ & $0.038(2)$ & $0.042(2)$ & $0.006(2)$ & $0.012(2)$ & $0.003(2)$ \\
\hline$O(5)$ & $4 e$ & $0.0730(4)$ & $0.1377(2)$ & $0.7315(3)$ & $0.048(3)$ & $0.063(3)$ & $0.054(3)$ & $-0.010(2)$ & $0.016(2)$ & $0.000(2)$ \\
\hline$O(6)$ & $4 e$ & $-0.1033(6)$ & $0.1486(3)$ & $0.6158(5)$ & $0.066(4)$ & $0.162(7)$ & $0.106(5)$ & $-0.004(4)$ & $-0.020(4)$ & $0.046(5)$ \\
\hline $\mathbf{O}(7)$ & $4 e$ & $-0.0891(6)$ & $0.0829(3)$ & $0.7181(5)$ & $0.109(5)$ & $0.077(4)$ & $0.165(6)$ & $-0.048(4)$ & $0.064(5)$ & $0.001(4)$ \\
\hline$O(8)$ & $4 e$ & $0.8975(5)$ & $0.2291(3)$ & $0.4529(4)$ & $0.069(4)$ & $0.101(4)$ & $0.096(4)$ & $-0.023(3)$ & $0.034(3)$ & $0.006(3)$ \\
\hline$O(9)$ & $4 e$ & $0.9109(6)$ & $0.2909(3)$ & $0.3506(4)$ & $0.067(4)$ & $0.164(6)$ & $0.077(4)$ & $0.022(4)$ & $0.013(4)$ & $0.049(4)$ \\
\hline$O(10)$ & $4 e$ & $0.9882(5)$ & $0.3093(2)$ & $0.5027(4)$ & $0.057(3)$ & $0.078(4)$ & $0.096(4)$ & $0.007(3)$ & $0.037(3)$ & $0.011(3)$ \\
\hline$C(1)$ & $4 e$ & $0.3296(6)$ & $0.3546(2)$ & $0.6472(4)$ & $0.041(3)$ & $0.041(3)$ & $0.040(3)$ & $-0.005(3)$ & $0.020(3)$ & $-0.007(3)$ \\
\hline$C(2)$ & $4 e$ & $0.4153(6)$ & $0.3605(2)$ & $0.7512(4)$ & $0.045(4)$ & $0.028(3)$ & $0.047(4)$ & $-0.008(2)$ & $0.015(3)$ & $-0.001(2)$ \\
\hline$C(3)$ & $4 e$ & $0.5516(6)$ & $0.3560(2)$ & $0.7767(5)$ & $0.050(4)$ & $0.045(4)$ & $0.058(4)$ & $-0.010(3)$ & $0.020(4)$ & $-0.007(3)$ \\
\hline$C(4)$ & $4 e$ & $0.6322(6)$ & $0.3562(3)$ & $0.8715(5)$ & $0.042(4)$ & $0.048(4)$ & $0.072(5)$ & $-0.003(3)$ & $0.008(4)$ & $0.001(3)$ \\
\hline$C(5)$ & $4 e$ & $0.5775(6)$ & $0.3612(3)$ & $0.9429(5)$ & $0.050(4)$ & $0.047(4)$ & $0.045(4)$ & $-0.004(3)$ & $-0.002(3)$ & $0.004(3)$ \\
\hline$C(6)$ & $4 e$ & $0.4441(6)$ & $0.3659(2)$ & $0.9179(4)$ & $0.053(4)$ & $0.051(4)$ & $0.038(4)$ & $0.003(3)$ & $0.010(3)$ & $-0.003(3)$ \\
\hline$C(7)$ & $4 e$ & $0.3603(5)$ & $0.3667(2)$ & $0.8223(4)$ & $0.042(3)$ & $0.027(3)$ & $0.036(3)$ & $-0.001(2)$ & $0.008(3)$ & $0.001(2)$ \\
\hline $\mathrm{C}(8)$ & $4 e$ & $0.2169(5)$ & $0.3755(2)$ & $0.7955(4)$ & $0.041(3)$ & $0.033(3)$ & $0.033(3)$ & $0.003(2)$ & $0.012(3)$ & $-0.003(2)$ \\
\hline $\mathrm{C}(9)$ & $4 e$ & $0.1360(6)$ & $0.3367(2)$ & $0.8180(4)$ & $0.039(3)$ & $0.038(3)$ & $0.031(3)$ & $0.002(3)$ & $0.006(3)$ & $-0.003(2)$ \\
\hline$C(10)$ & $4 e$ & $0.0043(6)$ & $0.3471(3)$ & $0.7918(5)$ & $0.046(4)$ & $0.051(4)$ & $0.053(4)$ & $0.006(3)$ & $0.015(3)$ & $-0.001(3)$ \\
\hline$C(11)$ & $4 e$ & $-0.0490(7)$ & $0.3963(3)$ & $0.7439(5)$ & $0.044(4)$ & $0.064(5)$ & $0.061(5)$ & $0.020(3)$ & $0.002(4)$ & $-0.002(4)$ \\
\hline$C(12)$ & $4 e$ & $0.0274(7)$ & $0.4347(3)$ & $0.7200(5)$ & $0.063(5)$ & $0.046(4)$ & $0.060(5)$ & $0.026(3)$ & $0.002(4)$ & $0.013(3)$ \\
\hline$C(13)$ & $4 e$ & $0.1595(6)$ & $0.4253(2)$ & $0.7456(4)$ & $0.062(4)$ & $0.040(3)$ & $0.041(4)$ & $0.000(3)$ & $0.014(3)$ & $0.000(3)$ \\
\hline$C(14)$ & $4 e$ & $0.1897(5)$ & $0.2808(2)$ & $0.8655(4)$ & $0.024(3)$ & $0.034(3)$ & $0.048(4)$ & $0.002(2)$ & $0.010(3)$ & $0.001(3)$ \\
\hline$C(15)$ & $4 e$ & $0.5064(6)$ & $0.2749(2)$ & $0.5377(4)$ & $0.054(4)$ & $0.037(3)$ & $0.049(4)$ & $-0.001(3)$ & $0.018(3)$ & $0.001(3)$ \\
\hline$C(16)$ & $4 e$ & $0.6412(6)$ & $0.2672(3)$ & $0.5751(5)$ & $0.048(4)$ & $0.053(4)$ & $0.055(4)$ & $0.011(3)$ & $0.008(3)$ & $0.004(3)$ \\
\hline$C(17)$ & $4 e$ & $0.7200(6)$ & $0.3097(3)$ & $0.5645(4)$ & $0.036(4)$ & $0.063(4)$ & $0.048(4)$ & $0.001(3)$ & $0.009(3)$ & $-0.005(3)$ \\
\hline$C(18)$ & $4 e$ & $0.6646(6)$ & $0.3591(3)$ & $0.5149(4)$ & $0.043(4)$ & $0.051(4)$ & $0.039(3)$ & $-0.011(3)$ & $0.013(3)$ & $-0.012(3)$ \\
\hline$C(19)$ & $4 e$ & $0.7420(6)$ & $0.4052(3)$ & $0.4996(5)$ & $0.038(4)$ & $0.081(5)$ & $0.052(4)$ & $-0.024(4)$ & $0.013(3)$ & $-0.008(4)$ \\
\hline$C(20)$ & $4 e$ & $0.6868(6)$ & $0.4518(3)$ & $0.4497(5)$ & $0.052(4)$ & $0.057(4)$ & $0.064(5)$ & $-0.021(3)$ & $0.026(4)$ & $-0.004(3)$ \\
\hline$C(21)$ & $4 e$ & $0.5480(6)$ & $0.4565(2)$ & $0.4092(4)$ & $0.049(4)$ & $0.045(3)$ & $0.040(4)$ & $-0.015(3)$ & $0.022(3)$ & $-0.007(3)$ \\
\hline$C(22)$ & $4 e$ & $0.4853(7)$ & $0.5020(3)$ & $0.3506(5)$ & $0.071(5)$ & $0.045(4)$ & $0.065(5)$ & $-0.020(3)$ & $0.036(4)$ & $-0.011(3)$ \\
\hline$C(23)$ & $4 e$ & $0.3520(7)$ & $0.5033(3)$ & $0.3101(5)$ & $0.071(5)$ & $0.043(4)$ & $0.072(5)$ & $-0.003(3)$ & $0.037(4)$ & $0.000(3)$ \\
\hline$C(24)$ & $4 e$ & $0.2805(6)$ & $0.4586(2)$ & $0.3294(5)$ & $0.045(4)$ & $0.041(4)$ & $0.065(4)$ & $0.001(3)$ & $0.023(4)$ & $0.001(3)$ \\
\hline$C(25)$ & $4 e$ & $0.4672(6)$ & $0.4123(2)$ & $0.4220(4)$ & $0.039(4)$ & $0.044(3)$ & $0.037(3)$ & $-0.006(3)$ & $0.014(3)$ & $-0.007(3)$ \\
\hline$C(26)$ & $4 e$ & $0.5285(5)$ & $0.3631(2)$ & $0.4777(4)$ & $0.037(3)$ & $0.047(3)$ & $0.030(3)$ & $-0.006(3)$ & $0.015(3)$ & $-0.004(2)$ \\
\hline$C(27)$ & $4 e$ & $0.4588(6)$ & $0.2110(2)$ & $0.7589(4)$ & $0.043(4)$ & $0.041(3)$ & $0.040(3)$ & $-0.001(3)$ & $0.016(3)$ & $0.002(3)$ \\
\hline$C(28)$ & $4 e$ & $0.5914(6)$ & $0.1971(3)$ & $0.7887(4)$ & $0.039(4)$ & $0.063(4)$ & $0.044(4)$ & $-0.003(3)$ & $0.008(3)$ & $0.011(3)$ \\
\hline C(29) & $4 e$ & $0.6282(6)$ & $0.1470(3)$ & $0.7571(5)$ & $0.044(4)$ & $0.069(5)$ & $0.059(4)$ & $0.015(3)$ & $0.023(4)$ & $0.018(3)$ \\
\hline$C(30)$ & $4 e$ & $0.5318(6)$ & $0.1115(3)$ & $0.6963(4)$ & $0.052(4)$ & $0.049(4)$ & $0.042(4)$ & $0.011(3)$ & $0.022(3)$ & $0.011(3)$ \\
\hline
\end{tabular}


Table 3. Continued.

\begin{tabular}{|c|c|c|c|c|c|c|c|c|c|c|}
\hline Atom & Site & $x$ & $y$ & $z$ & $U_{11}$ & $U_{22}$ & $U_{33}$ & $U_{12}$ & $U_{13}$ & $U_{23}$ \\
\hline $\mathrm{C}(31)$ & $4 e$ & $0.5629(8)$ & $0.0577(3)$ & $0.6641(5)$ & $0.083(5)$ & $0.050(4)$ & $0.064(5)$ & $0.020(4)$ & $0.040(4)$ & $0.006(3)$ \\
\hline$C(32)$ & $4 e$ & $0.4693(8)$ & $0.0243(3)$ & $0.6053(5)$ & $0.103(6)$ & $0.044(4)$ & $0.070(5)$ & $0.016(4)$ & $0.055(5)$ & $-0.002(3)$ \\
\hline$C(33)$ & $4 e$ & $0.3359(8)$ & $0.0426(3)$ & $0.5703(4)$ & $0.089(5)$ & $0.043(4)$ & $0.044(4)$ & $-0.010(4)$ & $0.036(4)$ & $0.001(3)$ \\
\hline$C(34)$ & $4 e$ & $0.234(1)$ & $0.0118(3)$ & $0.5016(5)$ & $0.129(8)$ & $0.048(4)$ & $0.063(5)$ & $-0.029(5)$ & $0.056(6)$ & $-0.014(4)$ \\
\hline$C(35)$ & $4 e$ & $0.1103(9)$ & $0.0327(3)$ & $0.4658(5)$ & $0.101(7)$ & $0.066(5)$ & $0.060(5)$ & $-0.039(5)$ & $0.039(5)$ & $-0.012(4)$ \\
\hline$C(36)$ & $4 e$ & $0.0848(7)$ & $0.0845(3)$ & $0.5027(5)$ & $0.066(5)$ & $0.072(5)$ & $0.054(4)$ & $-0.029(4)$ & $0.026(4)$ & $-0.003(4)$ \\
\hline$C(37)$ & $4 e$ & $0.3011(6)$ & $0.0952(2)$ & $0.6015(4)$ & $0.059(4)$ & $0.039(3)$ & $0.035(3)$ & $-0.003(3)$ & $0.022(3)$ & $0.003(3)$ \\
\hline$C(38)$ & $4 e$ & $0.4012(6)$ & $0.1294(2)$ & $0.6674(4)$ & $0.056(4)$ & $0.034(3)$ & $0.037(3)$ & $0.002(3)$ & $0.024(3)$ & $0.007(2)$ \\
\hline
\end{tabular}

Acknowledgments. We acknowledge financial support by the Zhejiang Provincial Technology Project Foundation of China (grant no. 2004C32088) and the Zhejiang Provincial Natural Science Foundation of China (grant no. 202137).

\section{References}

1. Wang, X. B.; Vittal, J. J.: Self-assembly of a 1D helical coordination polymeric lead(II) complex with $\mathrm{Pb}$...O(perchlorate)...Pb scaffoldings. Inorg. Chem. Commun. 6 (2003) 10741077.

2. Foreman, M. R. S. J.; Gelbrich, T.; Hursthouse, M. B.; Plater, M. J.: Hydrothermal synthesis and characterisation of lead(II) benzene-1,3,5-tricarboxylate [ $\mathrm{Pb}_{3} \mathrm{BTC}$ ] $-\mathrm{H}_{2} \mathrm{O}$ : a lead(II) carboxylate polymer. Inorg. Chem. Commun. 3 (2000) 234-238.

3. Cecconi, F.; Ghilardi, C. A.; Midollini, S.; Orlandini, A.: A new lead(II) inorganic-organic hybrid of the $P, P^{\prime}$-diphenylmethylene-diphosphinate ligand: synthesis and $X$-ray characterization of the $\left[\mathrm{Pb}\left(\mathrm{CH}_{2}\left(\mathrm{P}(\mathrm{Ph}) \mathrm{O}_{2}\right)_{2}\right)\right]$ complex. Inorg. Chem. Commun. 6 (2003) 546-548.

4. Bridgewater, B. M.; Parkin, G.: The synthesis and molecular structure of [TmPh]2Pb: a complex with an inverted coordination mode for the tris(2mercapto-1-phenylimidazolyl)hydroborato ligand. Inorg. Chem. Commun. 3 (2000) 534-536.

5. Ying, S. M.; Mao, J. G.; Yang, B. P.; Sun, Z. M.: Hydrothermal syntheses, characterizations and crystal structures of two new lead(II) diphosphonates with a layered and a microporous structure. Inorg. Chem. Commun. 6 (2003) 1319-1322.

6. Zhang, D. L.; Huang, C. H.; Xu, G. X.: Structural studies on rare earth carboxylates $I$. Studies on the crystal structure of tris(neopentanoato)- tris(neopentanoic acid)neodymium(III) dimer, $\left[\mathrm{NdA3}(\mathrm{HA})_{3}\right]_{2}$. Chin. J. Chem. 8 (1990) 529-535.

7. Hu, M. L.; Xiao, H. P.; Wang, S.; Li, X. H.: catena-Poly[t(1,10-phenanthroline- $x^{2} N, N^{\prime}$ )copper(II)]- $\mu$-(dihydrogenbenzene-1,2,4,5-tetracarboxylato) $-x^{2} \mathrm{O}^{1}: \mathrm{O}^{4}$. Acta Crystallogr. $\mathrm{C59}$ (2003) m454-m455.

8. Wang, M.; Xia, J.; Jin, L.; Cai, G.; Lu, S.: Crystal structure and luminescence property of novel three-dimensional network of $\left(\mathrm{Eu}_{2}(p-\mathrm{BDC})_{3}\right.$ (phen) $\left.)_{2}\left(\mathrm{H}_{2} \mathrm{O}\right)_{2}\right\}_{\text {n. }}$ J. Mol. Struct. 655 (2003) 443-449.

9. Paz, F. A. A.; Khimyak, Y.Z.; Bond, A. D.; Rocha, J.; Klinowski, J.: Synthesis and Characterization of a Novel Modular Cadmium-Organic Framework with Biphenyl-4,4'-dicarboxylate. Eur. J. Inorg. Chem. (2002) 2823-2828.

10. Zang, S. Y.; Yu, M. X.; Zhu, L. G.: Hydrothermal syntheses and crystal structures of two 4,4'-biphenyldicarboxylate copper(II) complexes with 1,10-phenanthroline. J. Mol. Struct. 699 (2004) 101-104.

11. Miao, Q; Hu, M. L.; Chen, F.: $\mu$-Aqua-bis( $\mu$-2'-carboxybiphenyl-2-carboxylato- $\left.x^{2} O ; O^{\prime}\right)$ bis[bis(2'-carboxybiphenyl-2-carboxylato- $\left.x O\right)(1,10$-phenanthroline- $\kappa^{2} N, N^{\prime}$ )-cobalt(II)]. Acta Crystallogr. E60 (2004) m1314-m1316.

12. Li, J. F.; Zhao, Y. J.; Li, X. H.; Hu, M. L.: Bis( $\mu$-hydrogen-5-sulfosalicylate)bis[aqua(1,10-phenanthroline)lead(II)]. Acta Crystallogr. E60 (2004) m1210-m1212.

13. Li, X. H.; Yang S. Z.: Bis( $\mu$-2-sulfonatobenzoato)bis[(1,10-phenanthroline)lead(II)] dihydrate. Acta Crystallogr. C60 (2004) m423-m425.

14. Sheldrick, G. M.: SHEIX-97. Programs for the Analysis of Crystal Structures (Release 97-2). University of Göttingen, Germany 1997.

15. Johnson, C. K.: ORTEP-II. A Fortran Thermal-Ellipsoid Piot Program. Report ORNL-5138. Oak Ridge National Laboratory, Oak Ridge, Tennessee, USA 1976. 